COMPLEJIDAD Y DELITOS SISTÉMICOS 
Rev. CAP Jurídica Central N. 4 - agosto 2019, ISSN 2550-6595

\title{
COMPLEJIDAD Y DELITOS SISTÉMICOS ${ }^{1}$
}

\author{
Omar Gabriel Orsi ${ }^{2}$ \\ Ministerio Público Fiscal (Argentina) \\ oorsi@mpf.gov.ar
}

\section{RESUMEN:}

Pese a los avances tecnológicos y las reformas procesales, la baja eficiencia en la investigación de los delitos sistémicos -en especial, los de cuello blanco- no pudo ser sustancialmente mejorada. El trabajo desarrolla el potencial de los modelos de la complejidad y de las metodologías formales para la resolución de este tipo de problemas.

\section{PALABRAS CLAVE:}

Modelos, complejidad, metodología, investigación penal, delitos sistémicos.

1 Artículo entregado por el autor el 15 de marzo de 2019 y aprobado el 25 de junio de 2019

2 Ministerio Público Fiscal (Argentina) oorsi@mpf.gov.ar Es doctor en Derecho por la Universidad de Salamanca, España y abogado por la Universidad de Buenos Aires, Argentina, con estudios en Ciencias Antropológicas en la misma universidad y de especialización en la Pontificia Universidad Católica de Argentina y en la Universidad de Chile. 
Rev. CAP Jurídica Central N. 4 - agosto 2019, ISSN 2550-6595

\section{ABSTRACT:}

Despite technological advances and procedural reforms, the low efficiency of systemic crimes investigation -specially white collar crimes- could not be substantially improved. The work develops the potential of the complexity models, and formal methodologies for solving this kind of problems.

\section{KEY WORDS:}

Models, complexity, methodology, criminal investigation, systemic crimes. 


\section{Introducción}

Pese a los esfuerzos, los sistemas penales de diversos países mantienen un comportamiento errático: mientras pueden tratar con rapidez y eficiencia cierto tipo de delitos -como los detectados en flagrancia- no ocurre lo mismo con otros -como los delitos sistémicos-. La dificultad para responder eficazmente ante los delitos sistémicos radica, entre otras razones, en que ellos no se encuentran claramente separados de la estructura de orden, como ocurre con la construcción simbólica del crimen organizado y el terrorismo; por el contrario, ellos se ubican en una zona preliminar, y no pueden ser separados tajantemente del devenir social lícito.

Esta característica incide notablemente sobre los sistemas de prevención, investigación y prueba de esta particular forma de delitos, y determina la imposibilidad de tratarlos con las mismas herramientas empleadas en los delitos de cuello azul. Los delitos sistémicos, como categoría que engloba todo delito del $\operatorname{poder}^{3} \mathrm{y}$ de productividad económica de cuello $_{\text {blanco }}{ }^{4}$, requiere de otras tecnologías y saberes, a los que la práctica judicial no parece adaptarse con facilidad. El objetivo del presente trabajo consiste en reflexionar sobre el potencial que presentan los modelos de tratamiento de la complejidad en la investigación y prueba de los delitos sistémicos.

3 Ruggiero. 2005.

4 Sutherland. 1949. 


\section{Aproximación al enfoque de la complejidad}

Los avances en el campo de la complejidad no surgieron repentinamente, sino que fueron madurando a lo largo del tiempo. Muchos de sus postulados y herramientas eran antiguos, pero se reformularon, combinaron y aceleraron en un momento en particular: la II Guerra Mundial y el posterior período de guerra fría, movidos por la necesidad de desarrollar potencial bélico.

Entre esas necesidades, se encontraban las de procesar e intercambiar información, de manera ágil y segura, entre las áreas operativas, de análisis y de comando, ante la eventualidad de un ataque masivo ${ }^{5}$, formas de detectar naves a grandes distancias, desarrollar nuevos materiales, acertar a un objetivo en movimiento, decodificar un mensaje encriptado, instalar un rumor, liberar energía de la materia, puntuar los ciclos de la economía o ejercer control sobre las masas en momentos críticos, entre otros. Este esfuerzo, impulsado por sectores vinculados a la defensa, involucró también al campo empresarial y académico, marcando así el rumbo de la agenda en los años posteriores.

Fue la necesidad -material e intelectual- la que llevó, tanto a aumentar la inversión, como a aguzar el ingenio. Y una de las formas de hacerlo fue reunir expertos de distintos campos para encontrar las respuestas -operativas-, que se estaban buscando. La estrategia no fue entonces sumar miradas de disciplinas consolidadas sobre un mismo tema, sino converger hacia una nueva forma de conocimiento para lidiar con problemas que, por su complejidad, ninguna de ellas había podido resolver satisfactoriamente.

BARAN. 1964; KLEINROCK. 2010. 
En el ámbito científico se produjo, por la misma época, una suerte de cambio copernicano. Encorsetadas por una visión mecanicista del mundo, y expurgados todo vestigio de holismo o animismo, diversas disciplinas comenzaban a advertir la estrechez de sus enfoques y a percibir su matriz común. En este ámbito, basado principalmente en los procesos de comunicación e información, confluyeron una serie de iniciativas, provenientes de distintos campos: la teoría clásica de sistemas, la verbal o semántica, la teoría de la información, la de juegos, la de decisión, la de autómatas, la de colas, la de conjuntos, la de gráficas o grafos, la de redes, la computarización-simulación, y la teoría cibernética, entre otras ${ }^{6}$. El enfoque, centrado en relaciones más que en entidades, permitió condensar así una serie de principios o herramientas útiles para toda clase de objetos, incluso aquellos de los que tratan las ciencias sociales.

La nueva perspectiva deja suficientemente en claro que no existen límites para la clase de modelo aplicado al estudio de la realidad, y que la aproximación, lejos de ser patrimonio de las denominadas ciencias duras, tuvo conspicuos representantes en las ciencias sociales ${ }^{7}$. También queda claro que las distintas ciencias enfrentaban un tipo de problema similar, y que éste podía separarse de la naturaleza del objeto: la salud mental, un circuito de telecomunicación, el funcionamiento físico del cerebro, la guía de un misil, la composición química de una sustancia, la estructura social o el lenguaje. Puede decirse que ese tipo de perspectiva no se circunscribe ni a un referente empírico determinado, ni a un saber en particular: se ubica, por así decir, en los límites de las disciplinas, en sus puntos de contacto.

6 Wiener. 1950; Von BertalanfFy. 1976.

7 BATESON. 1985. 


\section{Modelos de complejidad en ciencias sociales}

La corriente señalada en el punto anterior explora ciertas regularidades, independientes del tipo de objeto, de modo que acepta naturalmente que sus categorías no son lo abordado, sino un modelo, una ideación útil, en todo caso, para comprender u operar sobre determinados aspectos de la realidad ${ }^{8}$. Un modelo es una forma de lenguaje, una construcción humana de sentido. Los modelos se aproximan de cierto modo a las metáforas, los íconos o los símiles, ya que se espera que guarden algún tipo de relación con aquello que mentan.

Los modelos orientados al tratamiento de la complejidad se distinguen, sin embargo, por poseer un tipo específico de consistencia interna, debido al empleo de herramientas formales como la lógica o la matemática9 ${ }^{9}$ Puesto que manejamos sus pautas constructivas, podemos extender sus derivados, de modo que si el modelo resultó útil para tratar algunos aspectos de un objeto, es posible que lo sea para otros. La capacidad para establecer derivaciones formalmente confiables facilita, a su vez, la otra clave de bóveda de todo conocimiento operativo: la posibilidad de mapear el modelo contra la información aportada por las técnicas de construcción de datos. La modelización sirve, tanto para explorar las aristas relevantes de un problema, como en una etapa ulterior validar la opción escogida para tratarlo.

Los modelos pueden agruparse en cuatro clases, según la forma en que construyen su objeto de conocimiento, el tipo de inferencia

8 KORZYBSKI. 1951.

9 ReYNOSO. 2011. 
que apliquen y el propósito que persiguen, tal como se observa en la siguiente tabla ${ }^{10}$ :

\begin{tabular}{|c|c|c|c|}
\hline $\begin{array}{c}\text { Perspectiva del } \\
\text { objeto }\end{array}$ & $\begin{array}{c}\text { Tipo de } \\
\text { inferencia }\end{array}$ & Propósito & Modelo \\
\hline $\begin{array}{c}\text { Simplicidad } \\
\text { organizada }\end{array}$ & $\begin{array}{c}\text { Analítica, } \\
\text { deductiva, } \\
\text { determinista }\end{array}$ & Explicación & Mecánico \\
\hline $\begin{array}{c}\text { Simplicidad } \\
\text { desorganizada }\end{array}$ & $\begin{array}{c}\text { Estética, } \\
\text { abductiva, } \\
\text { indeterminista }\end{array}$ & Comprensión & Discursivo \\
\hline $\begin{array}{c}\text { Complejidad } \\
\text { desorganizada }\end{array}$ & $\begin{array}{c}\text { inductiva, } \\
\text { probabilista }\end{array}$ & Correlación & Estadístico \\
\hline $\begin{array}{c}\text { Complejidad } \\
\text { organizada }\end{array}$ & $\begin{array}{c}\text { Emergente, } \\
\text { determinista }\end{array}$ & $\begin{array}{c}\text { Descripción } \\
\text { estructural - } \\
\text { procesual }\end{array}$ & Sistémico \\
\hline
\end{tabular}

En este sentido, y a la hora de vincularse con los hechos, el procedimiento judicial se basa principalmente en modelos hermenéuticos o interpretativos, con el propósito de comprender un evento -conducta delictiva-, estructurado de manera simple, mediante la aplicación de los dictados de la lógica (deductiva) y de la experiencia (analogías). La aplicación de un modelo de estas características implica una forma de construir, procesar y validar información, lo cual explica el peso de la prueba testimonial y, en general, de los relatos.

Frente a ello, poca atención se ha prestado a los modelos formales específicamente orientados al tratamiento de la complejidad,

10 Basada en ReYnOSO. 2011. 
desarrollados por otros saberes, tal como es el caso de la teoría de redes. Para describirlo someramente, el enfoque de redes -como otros de este tipo- se caracteriza por desplazar el eje, desde los atributos de un ente a las relaciones entre ellos. Una tabla puede contener un conjunto de variables, dimensionadas conforme una serie de atributos, tal como es de utilización regular en la investigación en ciencias sociales. El siguiente es un ejemplo muy sencillo de tabla atributiva:

\begin{tabular}{|l|c|c|c|c|}
\hline & Universidad & Grado & Publicación & Investigación \\
\hline Matilda & Andina & Abogada & A & Salamanca \\
\hline Lautaro & Andina & Antropólogo & B & Random Inc. \\
\hline Rocío & Pompeu & Abogada & C & Salamanca \\
\hline Gino & Bologna & Sociólogo & A & Random Inc. \\
\hline
\end{tabular}

Esta representación permite exponer, de manera amigable, información sobre cada entidad: por ejemplo, que Rocío obtuvo el grado en Derecho en la Universidad Pompeu Fabra, que publicó el artículo "C" y que forma para de un grupo de investigación de la Universidad de Salamanca, y así sucesivamente.

La siguiente es una tabla relacional, construida a partir de la información anterior:

\begin{tabular}{|l|c|c|c|c|}
\hline & Matilda & Lautaro & Rocío & Gino \\
\hline Matilda & $\mathrm{X}$ & $\mathrm{X}$ & $\mathrm{XX}$ & $\mathrm{X}$ \\
\hline Lautaro & $\mathrm{X}$ & $\mathrm{X}$ & - & $\mathrm{X}$ \\
\hline Rocío & $\mathrm{XX}$ & - & $\mathrm{X}$ & - \\
\hline Gino & $\mathrm{X}$ & $\mathrm{X}$ & - & $\mathrm{X}$ \\
\hline
\end{tabular}

Esta tabla ya no se centra en los atributos, sino en las relaciones entre entidades. La tabla nos permite ver que Matilda es quien 
tiene más puntos en común con las restantes personas; que Lautaro y Gino, además de su relación con Matilda, se conectan entre sí; y que Rocío solo tiene contacto con Matilda, aunque más estrecho que el que poseen las restantes personas. Ahora bien, esa matriz de relaciones permite construir una representación gráfica, en forma de red. La representación -para otros datos, con once personas en lugar de cuatro- tiene el siguiente aspecto ${ }^{11}$ :

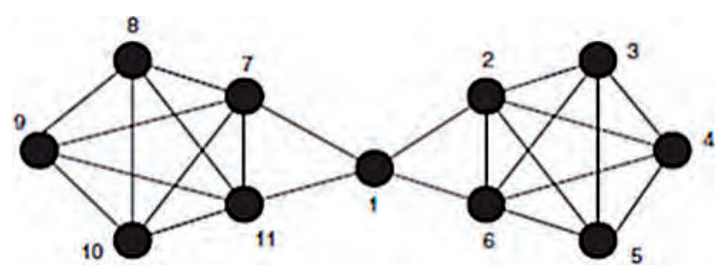

En este tipo de representaciones, las entidades -en el ejemplo, nuevamente, personas- se denominan nodos -representados por un punto- y sus relaciones vínculos o lazos -representados por una raya. Los datos, al ser transformados en un grafo, nos permiten percibirotrascosas: por ejemplo, quesi(1)noestuviera, tendríamos dos grupos fuertemente cohesionados, pero aislados entre sí.

La matriz correspondiente permite apreciar el poder visual del grafo anterior:

11 Extractado de BALLESTER et al. 2006. 
Rev. CAP Jurídica Central N. 4 - agosto 2019, ISSN 2550-6595

\begin{tabular}{|c|c|c|c|c|c|c|c|c|c|c|c|}
\hline & $\mathbf{1}$ & $\mathbf{2}$ & $\mathbf{3}$ & $\mathbf{4}$ & $\mathbf{5}$ & $\mathbf{6}$ & $\mathbf{7}$ & $\mathbf{8}$ & $\mathbf{9}$ & $\mathbf{1 0}$ & $\mathbf{1 1}$ \\
\hline $\mathbf{1}$ & $\mathrm{X}$ & $\mathrm{X}$ & - & - & - & $\mathrm{X}$ & $\mathrm{X}$ & - & - & - & $\mathrm{X}$ \\
\hline $\mathbf{2}$ & $\mathrm{X}$ & $\mathrm{X}$ & $\mathrm{X}$ & $\mathrm{X}$ & $\mathrm{X}$ & $\mathrm{X}$ & - & - & - & - & - \\
\hline $\mathbf{3}$ & - & $\mathrm{X}$ & $\mathrm{X}$ & $\mathrm{X}$ & $\mathrm{X}$ & $\mathrm{X}$ & - & - & - & - & - \\
\hline $\mathbf{4}$ & - & $\mathrm{X}$ & $\mathrm{X}$ & $\mathrm{X}$ & $\mathrm{X}$ & $\mathrm{X}$ & - & - & - & - & - \\
\hline $\mathbf{5}$ & - & $\mathrm{X}$ & $\mathrm{X}$ & $\mathrm{X}$ & $\mathrm{X}$ & $\mathrm{X}$ & - & - & - & - & - \\
\hline $\mathbf{6}$ & $\mathrm{X}$ & $\mathrm{X}$ & $\mathrm{X}$ & $\mathrm{X}$ & $\mathrm{X}$ & $\mathrm{X}$ & - & - & - & - & - \\
\hline $\mathbf{7}$ & $\mathrm{X}$ & - & - & - & - & - & $\mathrm{X}$ & $\mathrm{X}$ & $\mathrm{X}$ & $\mathrm{X}$ & $\mathrm{X}$ \\
\hline $\mathbf{8}$ & - & - & - & - & - & - & $\mathrm{X}$ & $\mathrm{X}$ & $\mathrm{X}$ & $\mathrm{X}$ & $\mathrm{X}$ \\
\hline $\mathbf{9}$ & - & - & - & - & - & - & $\mathrm{X}$ & $\mathrm{X}$ & $\mathrm{X}$ & $\mathrm{X}$ & $\mathrm{X}$ \\
\hline $\mathbf{1 0}$ & - & - & - & - & - & - & $\mathrm{X}$ & $\mathrm{X}$ & $\mathrm{X}$ & $\mathrm{X}$ & $\mathrm{X}$ \\
\hline $\mathbf{1 1}$ & $\mathrm{X}$ & - & - & - & - & - & $\mathrm{X}$ & $\mathrm{X}$ & $\mathrm{X}$ & $\mathrm{X}$ & $\mathrm{X}$ \\
\hline
\end{tabular}

Aunque en los ejemplos mencionamos personas y vínculos personales, cabe retener que puede tratarse de cualquier cosa y tipo de relación. Si seguimos en el plano humano, se analiza un conjunto mayor de vínculos dentro de una organización; la representación gráfica puede llegar a tener un aspecto similar al siguiente $^{12}$ :

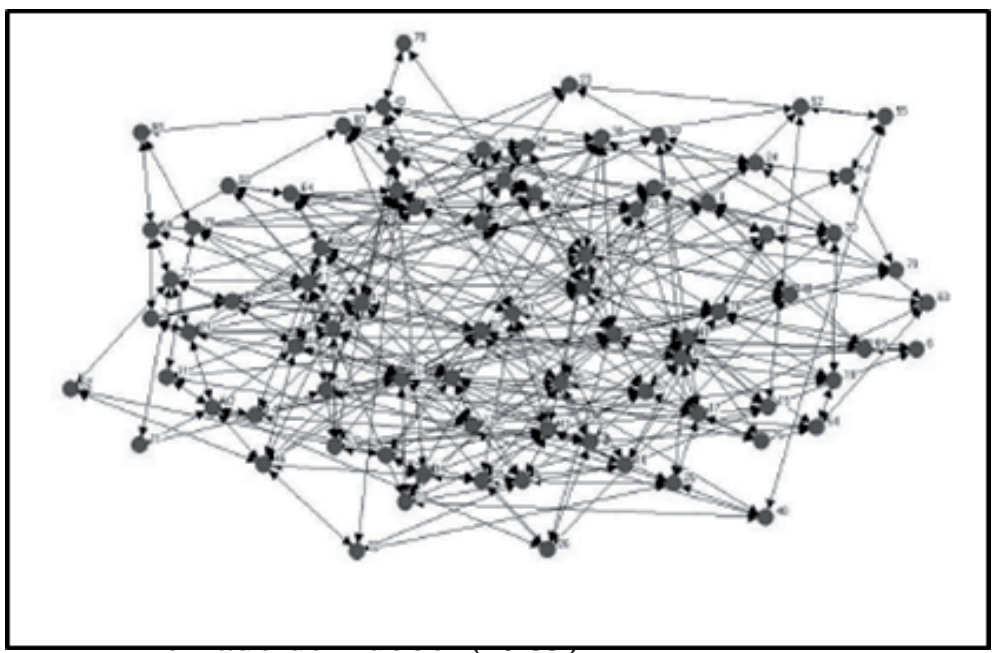


Al presentar la primera tabla, narramos debajo los atributos de una de las personas y pudimos haber continuado con las restantes: la tabla facilita esa exposición y permite "moverse" dentro de los datos, centrarse en algunos, contextualizar, comparar. Una vez dimensionadas las variables, los datos pueden procesarse por diversos métodos, tales como el estadístico. Del mismo modo, es imprescindible retener que el enfoque de matrices y grafos, va más allá de la representación gráfica de datos; ello se debe a que, reunidas determinadas condiciones, los modelos de redes -como ocurre con las matrices- muestran algunas propiedades formales comunes, que permiten inferir aspectos significativos para toda configuración de red.

Existen ciertamente muchos modelos, basados en el enfoque de redes. Conviene mencionar aquí una línea que se va abriendo paso de manera consistente, basada en las redes libre de escala, que son las que se adaptan a un número mayor de referentes empíricos, si son comparadas con la restante perspectiva -las redes aleatorias- ${ }^{13}$. En general, la mención apunta a destacar la capacidad del enfoque basado en redes libre de escala para aplicarse a objetos y datos de distinto tipo, con el potencial que ello implica en la investigación de cualquier clase de delito complejo.

13 BARABÁSI. 2003; REYNOSO. 2011. 


\section{Irrupción del modelo en el campo penal}

El ingreso del enfoque de la complejidad al sistema penal puede remontarse al auge, en la década de los ochenta del siglo pasado, de la problemática de las organizaciones delictivas y la consecuente demanda de seguridad ${ }^{14}$. El estudio jurídico de tales fenómenos conservaba, en ese entonces, los símiles tomados de los enfoques organizacionales, aplicados a asociaciones de objeto lícito, especialmente del mundo empresarial. Desde un polo ideal, puede decirse que estos enfoques partían de un arquetipo jerárquico, piramidal, que guardaba a su vez redundancia con la estructura normativa: ello se expresa incluso en la redacción de los delitos, al distinguir los integrantes de la asociación, tanto de sus jefes, como de otros roles preeminentes: los promotores, directores, fundadores, financiadores u organizadores ${ }^{15}$.

Si bien estos modelos fueron útiles, en su momento, para reducir las múltiples manifestaciones del fenómeno a un conjunto limitado de relaciones -como por ejemplo, la construcción de la figura de los capos de la mafia o los zares del narcotráfico-, no expusieron igual rendimiento a la hora de iluminar el funcionamiento de estructuras más flexibles, organizadas en función de objetivos concretos: fue en especial el impacto de una serie de atentados terroristas los que inclinaron el campo de investigación hacia estructuras celulares, en detrimento de otras formas de organización más vastas o contextualmente articuladas ${ }^{16}$.

\footnotetext{
14 Zaffaroni, E. 2001; Aniyar de CAstro. 2003; Grafe Arias. 2009; ORSI. 2007a.

15 Orsi. 2008a; Bueno Arús. 2009; Llobet Anglí. 2010; Wainwright. 2016.

16 Aparicio Díaz, y Ponte García. 2009.
} 
Como ocurriera con los anteriores modelos jerarquizados, el Derecho volvió a recurrir entonces a símiles tomados de las formas de organización del mundo lícito, en particular de su núcleo articulador, es decir, las estructuras económicas ${ }^{17}$. En éste también se había operado el desplazamiento, desde ciertas formas de producción -como el taylorismo y el fordismo- a otras -como el kanban o, luego, el modelo silicon valley-, con las consecuentes teorizaciones asociadas a las nuevas formas de organización -más flexibles, descentralizadas y adaptables a entornos cambiantes- y de gerenciamiento -con el procesamiento de información masiva e instantánea a través de ordenadores-. La tecnología necesaria también se encontraba madura: ella se produjo, en un derrotero para nada aleatorio, primero, en el terreno bélico para pasar luego a las fuerzas de seguridad, -como material de inteligencia especialmente orientado a la prevención-, y de allí al campo de trabajo del investigador judicial.

El impacto generado por ambos órdenes de fenómenos, sin perjuicio de sus características diferenciales, derivaron en la generación de un importante caudal de reflexiones, respecto de la capacidad del funcionamiento en red de organizaciones y personas ${ }^{18}$. En lo que toca al campo penal, la teoría de redes quedó así inicialmente asociada a un conjunto delimitado de fenómenos -integrado, básicamente, por distintos tipos de sujetos grupales clandestinos y violentos-; este proceso de síntesis, con sus más y sus menos, probó, sin embargo, el potencial del enfoque para la investigación de toda clase de delito complejo.

17 Albert. 1992; Atiles. 1994; ORSI. $2007 \mathrm{~b}$.

18 KREBs. 2001, 2004; Wu et al. 2014; PovedA CRIAdo. 2015. 
El estudio de atentados terroristas permitió comprobar la utilidad de los lazos débiles y de las estructuras flexibles para evitar la detección y ejecutar acciones de manera eficiente ${ }^{19}$. Por su parte, el enfoque de mundo pequeño -donde es posible navegar toda la red en pocos pasos- brinda una buena explicación sobre la capacidad de articular acciones, entre personas que no se conocen o que se conocen poco entre sí $^{20}$. Además, la capacidad de resiliencia de una red permite estudiar el modo en que determinadas organizaciones delictivas podían continuar actuando, aun luego de ser detectadas y parcialmente desmanteladas ${ }^{21}$.

De otro lado, varias de las medidas de red permitieron identificar diferentes funciones y posiciones de actores claves, cuya relevancia resulta de cierto modo análoga a los roles cumplidos dentro del entramado delictivo: en particular, esas aproximaciones permiten individualizar a personas con menor exposición y mayor poder de control sobre el flujo de acontecimientos, es decir, los roles propios de los organizadores, financistas y otras formas de intervención mediata en los hechos ${ }^{22}$. Finalmente, aunque sin agotar el listado, también permite detectar dentro de la red distintas formas de agrupación, caracterizadas según su grado de cohesión o tipo de relación o de control diferencial entre sus integrantes ${ }^{23}$.

19 Granovetter. 1973; Appadurai. 2006; BAdia i Dalmases. 2011.

20 Strogatz. 2003; SAgEMAN. 2000.

21 AyLing. 2009.

22 Carley, Krackhardt, y Lee. 2002; PaOli. 2004; Ballester et al. 2010; MARTí, y ZENOU. 2009; MORSELLI. 2009; BOUCHARD, y NGUYEN. 2010; Calderoni. 2011; Lauchs, Keast, y Chamberlain. 2011; SANTA AgReSte et al. 2015.

23

DunNE, y SHNEIDERMAN. 2013. 


\section{Adaptación del modelo a los delitos sistémicos}

Luego de la irrupción del enfoque reticular aplicado al crimen organizado y el terrorismo, el auge de los delitos de naturaleza económica impulsó las primeras iniciativas por adaptar a ellos dicho modelo. La tarea se vio, sin embargo, dificultada por el hecho de que, tanto el sesgo teórico, como la configuración de los dispositivos, no se encontraba adaptado a la complejidad diferencial del fenómeno. En efecto, el cambio de foco hacia los delitos sistémicos no sólo implica un mero desplazamiento de objeto: la aproximación, al articular de manera consciente el subsistema ilícito -oculto- con el lícito -visible-, acarrea una redefinición, tanto de los tipos de nodos, como de los vínculos relevantes, otrora reducidos a contactos -generalmente físicos o telefónicos- entre personas.

Aunque como hemos visto, no se trata de una exportación mecánica de las herramientas generadas para los delitos comunes, esta nueva aproximación también arroja algunos resultados alentadores. El enfoque sirve para iluminar los procesos de influencia y captura del estado, identificando los actores y nexos entre las esferas estatales y no-estatales ${ }^{24}$. De otro lado, también muestra utilidad para el estudio de los delitos de cuello blanco -como el blanqueo de capitales de origen delictivo-. El enfoque, debidamente adaptado, demuestra así buenas prestaciones a la hora de iluminar diversas formas de articulación, posición o relación social, propias del nuevo objeto, como la de influencia o mando, la disponibilidad estratégica de recursos, las relaciones cooperativas o independientes, la equivalencia de roles en función de similitudes estructurales, la intermediación entre partes poco

24 Garay Salamanca, y Salcedo-Albarán. 2012. 
conectadas o las formas de cohesión entre personas o grupos de personas $^{25}$. Como se observa, las categorías reticulares recién enunciadas resultan compatibles con sus símiles jurídicos de autoría -por determinación, dominio funcional, autoría mediata o paralela-, de cooperación -necesaria, accesoria- o de formas asociativas -roles dentro de una organización-, entre otras ${ }^{26}$.

En este sentido, y de las muchas aristas que presenta el tema, queda claro en todo caso que la adaptación del enfoque reticular a las distintas modalidades de delitos sistémicos, requiere de un trabajo específico. Por un lado, el mayor esfuerzo de adaptación -en comparación con los delitos extrasistémicos-, los cuales involucran primeramente a los insumos; por otro lado, en los casos de delitos clandestinos, nodos y vínculos quedan definidos de cierto modo, por su carácter natural (por ejemplo, personas y lugares, relación familiar y contacto telefónico). Pero los nodos de los delitos de cuello blanco -como el fraude al estado, el pago de coimas, el blanqueo de capitales y la financiación ilegal de partidos políticos-, pueden ser subcategorizados no solo en personas físicas, sino en cuentas, bancos, empresas o cualquier forma de persona jurídica o dispositivo formal o material de organización. Del lado de los lazos, estos también se multiplican: funciones dentro de una sociedad, representación de sociedades, titularidad de cuentas bancarias, intervención en operaciones financieras, comerciales, jurídicas, notariales, entre otros negocios jurídicos. Cada ítem mencionado admite además

25 Von Neumann, y Morgenstern. 1953; Hubbel. 1965; Coleman. 1972; Granovetter. 1973; Freeman. 1977; Burt. 1982; Marsden. 1984; Lozarez Colina. 1996; Monsalve. 2003; Roggero, y Silbertin-Blanc. 2008.

26 Miceli, Orsi y Rodríguez García. 2017. 
otras dimensiones: por ejemplo, la dirección de una transferencia o intensidad, de conformidad a un rango de montos.

Otro foco de complejidad radica en que, tanto la red, como las posiciones y funciones dentro de ella, tienden a solaparse; en otras palabras, y por tratarse de delitos sistémicos, lo usual es que sean los mismos dispositivos de organización y estructura de la esfera lícita los empleados en la comisión de delitos. Es por ello relevante que los modelos aplicados a estos fenómenos, en lugar de emplear variables naturales, personales o de sentido común, se construyan en función de las reglas de orden del sistema al cual son aplicados. La siguiente tabla expone un ejemplo de adaptación de las señales de alerta, establecidas por una institución para prevenir casos de lavado $^{27}$ y su conversión a un valor significativo para la teoría de redes $^{28}$.

27 GAFISUD. 2012.

28 Tomado de Micelli, Orsi y Rodríguez García. 2017. 
Rev. CAP Jurídica Central N. 4 - Agosto 2019, ISSN 2550-6595

\begin{tabular}{|c|c|}
\hline Señales jurídicas de alerta & Señales reticulares de alerta \\
\hline $\begin{array}{l}\text { Movimientos de dinero no acordes al } \\
\text { perfil económico. }\end{array}$ & Atributo inusual de lazo \\
\hline $\begin{array}{l}\text { Las personas que reciben las remesas no } \\
\text { tienen relación con los migrantes. }\end{array}$ & $\begin{array}{l}\text { Atributo de un nodo o clase nodo } \\
\text { comparado con atributo de otro nodo o } \\
\text { clase de nodos }\end{array}$ \\
\hline $\begin{array}{c}\text { Creación de gran cantidad de empresas de } \\
\text { papel, sin tener la logística ni los medios } \\
\text { necesarios para su funcionamiento. }\end{array}$ & Atributo inusual de un grupo de nodos \\
\hline $\begin{array}{c}\text { Compañías que no registran proveedores } \\
\text { locales de las mercancías exportadas. }\end{array}$ & Atributo inusual de un grupo de nodos \\
\hline $\begin{array}{l}\text { Productos exportados que no serían } \\
\text { originarios del país A. }\end{array}$ & Atributo inusual de un grupo de nodos \\
\hline $\begin{array}{l}\text { Precios de comercialización en el exterior } \\
\text { son superiores a los del mercado local. }\end{array}$ & $\begin{array}{l}\text { Atributo de un grupo de nodos comparado } \\
\text { con atributo de otro grupo de nodos }\end{array}$ \\
\hline $\begin{array}{l}\text { Exportación de un bien al país productor } \\
\text { del mismo }\end{array}$ & $\begin{array}{l}\text { Atributo inusual de un lazo o grupo de } \\
\text { lazos }\end{array}$ \\
\hline $\begin{array}{l}\text { Estratificación de fondos para evitar } \\
\text { controles. }\end{array}$ & $\begin{array}{l}\text { Atributo específico de un nodo o grupo } \\
\text { de nodos }\end{array}$ \\
\hline $\begin{array}{l}\text { Actividad económica declarada diferente } \\
\text { a la actividad económica realizada. }\end{array}$ & $\begin{array}{l}\text { Atributo real de un nodo o grupo de nodos } \\
\text { diferente a lo declarado }\end{array}$ \\
\hline $\begin{array}{c}\text { Transferencias enviadas y recibidas no } \\
\text { tienen relación con las declaradas }\end{array}$ & $\begin{array}{l}\text { Atributo de un lazo o conjunto de lazos } \\
\text { comparado con atributos declarados }\end{array}$ \\
\hline $\begin{array}{l}\text { Información y documentación presentada } \\
\text { incompleta. }\end{array}$ & $\begin{array}{l}\text { Atributo específico de un nodo o grupo } \\
\text { de nodos }\end{array}$ \\
\hline $\begin{array}{l}\text { Giro de cheques por montos altos } \\
\text { cobrados en el exterior. }\end{array}$ & $\begin{array}{l}\text { Atributo específico de un lazo o grupo } \\
\text { de lazos }\end{array}$ \\
\hline $\begin{array}{c}\text { Los pagos provienen de países diferentes } \\
\text { al que se exportaron los productos. }\end{array}$ & Atributo específico de un grupo de nodos \\
\hline $\begin{array}{l}\text { Transferencias a otros países donde no se } \\
\text { realizó la actividad comercial. }\end{array}$ & $\begin{array}{l}\text { Atributo específico de un lazo o grupo } \\
\text { de lazos }\end{array}$ \\
\hline $\begin{array}{c}\text { Circulación de dólares en billetes en de } \\
\text { alta denominación registrados en listas } \\
\text { reservadas. }\end{array}$ & $\begin{array}{l}\text { Atributo específico de un lazo o grupo } \\
\text { de lazos }\end{array}$ \\
\hline $\begin{array}{l}\text { Canje de billetes de alta denominación } \\
\text { por billetes de baja denominación. }\end{array}$ & $\begin{array}{l}\text { Atributo específico de un lazo o grupo } \\
\text { de lazos }\end{array}$ \\
\hline
\end{tabular}




\section{Uso de modelos en la fase de investigación}

Lo visto hasta aquí nos lleva a resaltar la importancia del momento metodológico del proceso de investigación, como articulador entre los modelos de la complejidad y las técnicas que ellos utilizan, cuestión relevante no sólo por tratarse de un requisito inescindible de la aplicación de ese tipo de modelos, sino por su falta de desarrollo acabado en el campo penal.

Es posible postular que, en el estado actual, la metodología de investigación penal ha perdido espesor por dos razones. De un lado, porque las técnicas especiales aplicadas a los denominados delitos complejos -como la denuncia anónima, el arrepentido, el agente encubierto, el testigo protegido, entre otras- no requieren de mecanismos de inferencia demasiado sofisticados, sino que ellas se producen, en última instancia, bajo la forma de relato, como el testimonio o la confesión ${ }^{29}$. Del otro lado, porque las nuevas herramientas de procesamiento de la información, además de no emplearse de manera sistemática, quedan reducidas a su aspecto instrumental. Esta última situación puede llevar a que el director jurídico del proceso de investigación se desentienda de ellas y, eventualmente, entregue su ejecución a otros expertos, usualmente ubicados en una institución o estructura distinta, en el entendimiento de que solo se trata de apretar un botón.

Bajo esta doble constricción, los modelos para abordar la complejidad pierden gran parte de su potencial, si es que no se encuentran acompañados de una estructura operativa y de una dirección metódica desde los inicios de la investigación: en otras palabras, no se puede suplir, sin pérdida, por un informe técnico

29 Del Rosal Blasco. 2009; SOtOmayor Acosta. 2010. 
o un peritaje, aunque sean esas sus formas de materialización procesal.

Es necesario así prestar debida atención a los diferentes momentos que conforman la metodología de investigación de esta especial clase de delitos, por las complejidades que presenta. En efecto, los casos simples suelen caracterizarse por la comunicación al investigador de una hipótesis suficiente sobre el conflicto. Los de investigación compleja, en cambio, por presentar un indicio fragmentario del evento delictivo, que requiere por ello de una construcción secuenciada de la hipótesis del caso. El paso del indicio al caso supone así una forma de inferencia específica, basada en conocimiento imperfecto: si los indicios sugieren una configuración general similar a otra conocida, podemos, con los recaudos del caso, tratarla como si fuera igual ${ }^{30}$.

La formulación inicial del caso resulta, pues, un momento importante en las investigaciones complejas. Si la hipótesis ha sido deficientemente formulada -por falta de consistencia interna-, si es estrecha -por circunscribirse a una sola de las posibilidades- o tendenciosa -por excluir vías de indagación de manera arbitraria o dirigir imputaciones aviesas-, ello introducirá un sesgo altamente nocivo en los pasos posteriores. De la otra mano, no toda conjetura determina mecánicamente la amplificación de las hipótesis: lo que puede ser norma en la actividad científica, no lo es en el terreno jurídico penal, donde el alcance y profundidad de la tarea se encuentra encorsetada por los principios que rigen el debido proceso. Las hipótesis deben ser amplias, pero sustentables y razonables, con límite en las

30 Piaget. 1969; Peirce. 1970; James. 1984; Bateson.1985;Mandelbrot. 1987; DARWIN. 2003. 
conexiones excepcionales, las inferencias meramente posibles o las imputaciones tendenciosas.

Modelizar el problema que suponen las hipótesis es, pues, otro momento importante de la investigación: las hipótesis son, entonces, el marco general, el cual debe anticipar la configuración del fenómeno, cuya modelización opera como guía de la investigación $^{31}$. La modelización es una operación conceptual que permite dimensionar el objeto, mediante el análisis de sus componentes principales, de modo de derivar variables e indicadores -atributivos o relacionales- que admitan alguna forma de mapeo con la realidad, en el caso jurídico, a través de los medios de prueba ${ }^{32}$.

Para el éxito de esta operación resulta relevante atender, en primer lugar, al esquema que propone el tipo delictivo, como elementos indiciarios que anticipan la posible existencia del delito: los vinculados a la lesividad y la conducta que realiza el tipo o los elementos, materiales o normativos, jurídicamente indispensables. Junto a esas variables o dimensiones primarias pueden ubicarse otras accesorias, como las formas eventuales de intervención en el hecho -por ejemplo, instigación, autoría mediata, participaciones secundarias-, o las fases de preparación y agotamiento del delito, entre otras. El modelo normativo debe a su vez ser complementado con otras regularidades, provenientes de su forma de realización, ya que las conductas y elementos previstos de manera abstracta en la norma suelen concretarse a través de formas regulares de ejecución, o modos comisivos:

\footnotetext{
31 ORSI. 2018.
}

32 Galtung. 1966; Samaja. 1993; Axelrod. 2004; Armatte. 2006; Arroyo Méndez y Hassan Collado. 2007. 
por caso, ciertas modalidades de blanqueo de capitales o de fraude impositivo se caracterizan, en determinados lugares y épocas, por el uso de operaciones financieras, entidades, plazas y personas jurídicas específicos, que no responden a la racionalidad o regularidad del tipo de negocio que le sirve de causa aparente. Estos aspectos conllevan -como hemos visto en el punto previouna mayor articulación con la realidad que los componentes normativos, incrementando la complejidad de la tarea.

Las variables e indicadores inferidos de ambas formas modélicas -normativas y extra normativas- priorizan entonces algunos aspectos del fenómeno, orientando la búsqueda de prueba y facilitando el mapeo de la información adquirida. Si el núcleo de la inferencia de hipótesis es anticipar analógicamente la o las configuraciones probables, la modelización del caso persigue dos objetivos básicos:

a) Operativizar el criterio por el cual se han admitido hipótesis alternativas o, lo que es lo mismo, dimensionar los elementos esenciales de los componentes de una clase; $y$,

b) Hacer lo propio con aquellos puntos que se encuentran, de modo excluyente, en cada hipótesis.

La fase de modelización inicial prefigura así los núcleos que deben ser acreditados o descartados durante la investigación. La ejecución de la prueba sobre los tópicos comunes es siempre relevante, pues hace a todas las hipótesis en danza; la de los dirimentes juega un rol aún más importante: descartar alternativas inadecuadas y, con ello, simplificar el marco de búsqueda y las medidas probatorias subsecuentes. Esta función de descarte es 
primordial en el reajuste de las hipótesis provisorias conforme la realidad que va revelando el caso. Es por ello crítico que el descarte opere con certeza ${ }^{33}$.

Otro momento importante es la delimitación del campo en el que se inscribe la conducta. La delimitación del campo de búsqueda es una operación, basada en el modelo exploratorio, que permite identificar los ámbitos o lugares que cobijan información relevante para la investigación. Mientras que la modelización racionaliza el fenómeno en función de las hipótesis típicas y las regularidades conductuales, la delimitación del campo permite iluminar los distintos contextos en que se inscribe la conducta.

Como se observa, es imprescindible que los investigadores dispongan de una reconstrucción previa y proactiva de las conductas y contextos propios de los conflictos de su competencia, con sus reglas y pautas de orden. $\mathrm{Y}$ es fundamental que ese conocimiento previo no quede circunscripto a la experiencia personal de los operadores, sino que se traduzca en dispositivos operativos. Es necesario entonces, tal como hemos visto en el punto previo con el ejemplo de las tipologías sobre blanqueo, que los modelos exploratorios del campo jurídico se objetiven también en un dispositivo informático especialmente adaptado para el almacenamiento y entrecruzamiento de información.

Una vez que comienza a incorporarse la información, otro momento crucial de la investigación es detectar los patrones subyacentes en el conjunto de datos. La inferencia de hipótesis, la modelización del caso y la delimitación del campo permiten descartar alternativas de imposible cumplimiento, prefigurar 
Rev. CAP Jurídica Central N. 4 - agosto 2019, ISSN 2550-6595

los caminos que debe seguir la investigación y puntuar, dentro de ellos, algunos elementos, relaciones y entornos críticos. La búsqueda de patrones, por su parte, apunta a inferir regularidades en el objeto, según la heurística aplicada al caso ${ }^{34}$.

34 HANSON. 1985; WATANABE. 1985; SCHÜRMANN. 1996. 


\section{Uso de modelos como herramienta probatoria}

Como ocurre con la etapa de investigación, en este caso también se advierte un repliegue del espesor de la etapa probatoria, en función del peso adquirido por los relatos, en especial desde el pasaje de los sistemas penales de la escritura a la oralidad. Por diversas razones -derivadas de la primacía dada a la voluntad, tal como se observa en el derecho a la acción y en la negociaciónlos relatos no requieren de operaciones alambicadas a la hora de motivar una resolución, puesto que ellos siguen la gramática empleada por los propios jueces y jurados ${ }^{35}$.

Pero más allá de toda consideración al respecto, lo concreto es que los delitos sistémicos parecen requerir de un esfuerzo mayor. En primer lugar, porque resulta difícil obtener testimonios o confesiones confiables, en particular cuanto mayor es el poder de la organización, o más radicalizada la puja entre factores de poder. En segundo lugar, porque, en esos contextos, las voluntades pueden ser dirigidas para disputar mercados o esferas de poder, amplificando el problema. En tercer lugar, porque al carecer de exhaustividad, pueden apuntar a responsabilidad menores, sin comprometer los núcleos reproductivos de los delitos económicos. Finalmente, porque su propio modo de realización sugiere otra fuente de datos complementaria, más rica y confiable: si se trata de conductas complejas, coordinadas, con participación múltiple e impacto institucional o económico, lo esperable es que dejen un rastro instrumental o documental calificado.

En todo caso, es claro que no existe oposición entre ambos sistemas, de modo que pueden utilizarse de manera conjunta, para potenciar sus virtudes y compensar sus debilidades.

35 ORSI. 2018. 


\begin{tabular}{|c|c|c|}
\hline \multirow{2}{*}{$\begin{array}{c}\text { Inferencia } \\
\text { basada } \\
\text { en } \\
\text { relatos }\end{array}$} & \multirow{3}{*}{$\begin{array}{l}\text { EJE DEL SISTEMA DE INFERENCIA } \\
\text { - Procesamiento exhaustivo de la prueba } \\
\text { individual para interpretarla } \\
\text { - Ensamble de la prueba: } \\
\text { - Por la configuración más razonable } \\
\text { (Lógica y experiencia) } \\
\text { - Excluyendo configuraciones } \\
\text { menos razonables }\end{array}$} & \multirow{3}{*}{$\begin{array}{l}\text { Puntos fuertes y débiles: } \\
\text { - Punto fuerte: Se apoya principal- } \\
\text { mente en el valor que aporta } \\
\text { cada prueba } \\
\text { - Punto débil: la reconstrucción de } \\
\text { la totalidad no se apoya en el } \\
\text { análisis exhaustivo del conjunto de } \\
\text { datos, mapeados contra un modelo } \\
\text { formal }\end{array}$} \\
\hline & & \\
\hline $\begin{array}{l}\text { en } \\
\text { relatos }\end{array}$ & & \\
\hline
\end{tabular}

\begin{tabular}{|c|c|c|}
\hline $\begin{array}{c}\text { Inferencia } \\
\text { basada en } \\
\text { información } \\
\text { objetiva }\end{array}$ & $\begin{array}{l}\text { EJE DEL SISTEMA DE INFERENCIA } \\
\text { - Procesamiento exhaustivo del conjunto } \\
\text { de datos para inferir patrones }\end{array}$ & $\begin{array}{l}\text { Puntos fuertes y débiles: } \\
\text { Mapeo de los patrones contra modelos: } \\
\text { - Por consistencia interna del modelo } \\
\text { - Excluyendo modelos alternativos } \\
\text { por inconsistencia }\end{array}$ \\
principalmente en el valor de la \\
prueba aislada
\end{tabular}

La ventaja comparativa del enfoque basado en información objetiva es que brinda una buena plataforma integrar datos de fuente y lenguaje diverso -que es donde radica gran parte de la complejidad de los delitos sistémicos-, de modo de facilitar su clasificación, orden e interpretación. Esos datos, insistimos, no se expresan en el mismo código del operador judicial, tal como ocurre con los testimonios o las confesiones, lo cual complica la apreciación directa de la prueba. Para validarlos será necesario reconstruir los patrones que anidan en esa información y mapearlos contra un modelo, de manera dinámica, mediante una metodología confiable ${ }^{36}$. 
Ahora bien, uno de los principales escollos radica en si esas construcciones, además de su consistencia interna, pueden ser razonablemente traducidos al lenguaje judicial, o si son, ora irreductibles, ora la empresa de tal dificultad que la desalienta. En este sentido, y tal como hemos visto para la conducta, también puede señalarse la existencia de una analogía, para validar o descartar la hipótesis del caso, entre ambas formas modélicas -redes y jurídica- ${ }^{37}$. En efecto, existe hoy día un razonable acuerdo sobre la importancia adquirida por la reconstrucción indiciaria dentro del sistema probatorio ${ }^{38}$. Este esquema coloca el eje en el factor relacional: el valor probatorio proviene aquí de la integración del conjunto de indicios. La operación, al ser integral, abarca cualquier elemento pertinente y útil: el diferencial radica en la integración de todos los elementos en un contexto coherente, movimiento que reafirma tanto el valor individual de cada prueba como el de la reconstrucción o explicación del caso.

Como se observa, el sistema de prueba indiciaria coincide estructuralmente con la metodología propuesta para la investigación y acreditación de los delitos complejos, ya que ambas son, en esencia, operaciones de mapeo: al requisito de pertinencia y acreditación inequívoca de los indicios -graves y precisos-, se suma la necesidad de adecuación no contradictoria dentro un contexto concreto -concordantes-. La ventaja del

SÁNCHEZ. 2016.

Alexy. 1989.

38 Silva Melero. 1944; Juanes Peces. 1989; Bajo Fernández. 1991; Asencio Mellado. 1992; Atienza. 1997; Russo y Abet. 2001; Pastor Alcoy. 2003; Parra Quijano. 2007; GuzMán. 2011; SuÁrez. 2011; CoRdón Aguilar. 2012. 
sistema propuesto radica en que, en este caso, el contexto a) es un modelo formal dotado de consistencia interna, b) que admite contrastación empírica mediante una metodología controlable, y c) que permite el descarte de modelos alternativos.

El que ambas formas de inferencia y prueba compartan una base común tiene, pues, importantes consecuencias. De un lado, reafirma el valor jurídico del método propuesto y la posibilidad de traducirlo a un lenguaje común, que fomente el trabajo transdisciplinario o su valoración en juicio. De otro lado, pone en evidencia que un método de esas características excede el alcance propio del peritaje: en realidad, se trata de una herramienta para construir, contrastar y mejorar el propio método de inferencia y validación judicial.

Existe, finalmente, una prestación adicional del enfoque reticular, vinculado a su potencial persuasivo y su aptitud para adaptarse al sistema de audiencias orales, en las que suelen resolverse algunos aspectos centrales del proceso. Suele vincularse a las audiencia con los discursos argumentativos, construidos en un registro expresivo y emotivo, destinado a generar adscripción y empatía: sin embargo las audiencias incorporan día a día otros recursos, como imágenes, filmaciones, gráficas o representaciones visuales de diverso tipo (tal como ocurre en paralelo en la enseñanza o en el dictado de conferencias, con el empleo de presentaciones o infografías) por su capacidad de apelar a un sentido primordial del ser humano para relacionarse y construir su entorno. Esa apelación al componente visual se relaciona directamente con la capacidad que poseen ciertas herramientas —como tablas, matrices, conjuntos, grafos, gráficos, funciones, curvas normales, organigramas, diagramas de flujo, entre otros - de representar o evocar información 
compleja de manera aprehensible, extremo que resulta más dificultoso cuando se apela, exclusivamente, al lenguaje oral o escrito ${ }^{39}$.

Así, aun cuando la faceta constructiva del procesamiento y análisis de información se produce mediante un lenguaje distinto al de los jurados o jueces, esta dificultad se revierte a la hora de exponer el resultado, en particular si no se utilizan imágenes de manera fragmentaria, con sentido emotivo. En efecto, las representaciones a que aquí aludimos no sólo operan positivamente por el hecho de esquematizar aquello que es complejo, sino porque, a diferencia de lo que ocurre con un testimonio o un documento, implican siempre su inscripción dentro de un patrón de orden: la redundancia entre la descripción y la regla refuerza así el valor persuasivo del medio, esto es, el poder visual de la expresión gráfica. La clasificación ordenada de las partes de una configuración, en sí valiosa para cualquier observador externo, se verá reforzada en la medida en que esa construcción haya sido generada mediante procedimientos teóricos y metodológicos fundados.

39 BRANDES, KENIS, y RAAB. 2005. 


\section{Consideraciones finales}

La comunicación a través de dispositivos es algo inherente al modo de vida actual. Muchas de esas comunicaciones nos son impuestas, como las operaciones bancarias o los trámites administrativos. Existe además un enorme cúmulo de información que entregamos voluntariamente, en particular quienes no vivieron, o no han prestado debida atención, al proceso histórico que llevara al desarrollo tecnológico ${ }^{40}$. Un evidente peligro, claro está, en especial porque la capacidad de almacenamiento -siendo antes un problema- crece ahora de manera exponencial, mientras se gestan, a la par, ordenadores cada vez más veloces.

Pero también una oportunidad, ante ataques graves, si es que se hace un uso prudente y dentro de estrictos marcos de legalidad. Dentro de ese marco -insistimos, de derecho penal mínimo, de estricto apego a su condición de ultima ratio y de respeto a rajatabla de las garantías que rigen el debido proceso- creemos que es posible aplicar a las investigaciones penales complejas algunas de las herramientas elaboradas por otros saberes. La alta eficiencia que puede aportar esta estrategia a la investigación de los delitos sistémicos no es sólo un valor de por sí, sino que su empleo en esos casos puede ayudar a equilibrar, de algún modo, la falta de equidad que caracteriza a la persecución penal, casi siempre centrada en determinado tipo de delitos -los de cuello $a z u l$ - o en otros que dicta la coyuntura -peleas de poder-.

Pese a que, como habrá advertido el lector, no se trata ni de teorías nuevas, ni de herramientas desconocidas para el sistema penal, su aplicación se encuentra por demás demorada y reducida

BARAN. 1964. 
a conflictos simples. Demora paradójica para los discursos de ley y orden, de maximización de la herramienta penal y de inocuización -física y simbólica- del imputado, pues ellos se siguen solazando en métodos y recursos toscos, que cuesta poner a consideración del resto de la comunidad académica. Lejos de la dificultad teórica o de los costos -que no son muchos- que pudiera insumir la aplicación del enfoque de la complejidad a los delitos sistémicos, podemos conjeturar que la reticencia obedece a razones estructurales, a constricciones que escapan a las consideraciones de este trabajo, y que se encuentran inexorablemente imbricadas con la selectividad y los efectos materiales concretos -no supuestos- de los dispositivos de criminalización. 
Rev. CAP Jurídica Central N. 4 - agosto 2019, ISSN 2550-6595

\section{BIBLIOGRAFIA}

AlBert, M. Capitalismo contra capitalismo. Buenos Aires, Paidós, 1992.

ALEXY, R. Teoría de la argumentación jurídica. La teoría del discurso racional como teoría de la fundamentación jurídica. Madrid, Centro de Estudios Políticos y Constitucionales, 1989.

ANIYAR DE CASTRO, L. "La sombra de 'El Padrino'. Concepto criminológico del delito organizado", Revista Brasileira de Ciências Criminais. N. ${ }^{\circ} 42,2003$.

APARICIO DÍAZ, L., y PONTE GARCÍA, M. "La célula terrorista de Madrid como célula independiente: un análisis jurídico del 11-M", Cuadernos de Política Criminal. N. ${ }^{\circ}$ 99, 2009.

APPADURAI, A. Fear of Small Numbers. An Essay on the Geography of Anger. London, Duke University Press, 2006.

ARMATTE, M. "La noción de modelo en las ciencias sociales", Empiria Revista de Metodología de Ciencias Sociales. N. ${ }^{\circ} 11,2006$.

ARROYO MÉNDEZ, M. y HASSAN COLLADO, S. "Simulación de procesos sociales basada en agentes software", Empiria Revista de Metodología de Ciencias Sociales. N. ' 14, 2007. 
ASENCIO MELLADO, J. M. "Presunción de inocencia y prueba indiciaria”, VV. AA. Los principios del proceso penal. Madrid, Consejo General del Poder Judicial, 1992.

ATIEnZA, M. Las razones del Derecho. Teorías de la argumentación jurídica. 2. ${ }^{\text {a }}$ edición, Madrid, Centro de Estudios Constitucionales, 1997.

ATILES, A. "La empresa-red: un modelo de división del trabajo entre empresas", Papers Revista de Sociología. v. 44, 1994.

AXElROD, R. La complejidad de la cooperación. Modelos de cooperación y la colaboración basada en los agentes. Buenos Aires, Fondo de Cultura Económica, 2004.

AYLING, J. "Criminal organizations and resilience". International Journal of Law, Crime and Justice. Vol. 37, 2009.

BADIA I DALMASES, F. "Entender las redes terroristas del mundo pequeño: hacia un mundo post-Al Qaeda", Documentos CIDOB. N. ${ }^{\circ}$ 7, 2011.

BAJO FERNÁNDEZ, M. "Presunción de inocencia, presunción legal y presunción judicial o prueba de indicios", Diario La Ley. N. ' $1,1991$.

BAllester, C., CAlvó-ARMEngOl, A., and ZENOU, Y. "Who's Who in Networks. Wanted: the Key Player", Econometrica. Vol. 74, 2006. 
BARABASI, A. L. Linked: How Everything Is Connected to Everything Else and What It Means. New York. Plume Books, 2003.

BARAN, P. "On Distributed Communications Networks", Communications Systems. IEEE Transactions, Vol. 12, N. ${ }^{\circ} 1,1964$.

BAteson, G. Pasos hacia una ecología de la mente. Buenos Aires, Lohlé, 1985.

BOUCHARD, M., and NGUYEN, H. "Patterns of Youth Participation in Cannabis Cultivation”, Journal of Drug Issues. Vol. 40, 2010.

BRANDES, U., KENIS, P., y RAAB, J. "La explicación a través de visualización de redes", Revista Redes Revista Hispana para el Análisis de Redes Sociales. N. ${ }^{\circ}$ 5, v. 9, 2005.

BUENO ARÚS, F. Terrorismo: algunas cuestiones pendientes. Valencia, Tirant lo Blanch, 2009.

BURT, R. S. Toward a Structural Theory of Action: Network Models of Social Structure, Perception and Action. New York, Academic Press, 1982.

Calderoni, F. "Where Is the Mafia in Italy? Measuring the Presence of the Mafia across Italian Provinces", Global Crime. N. ${ }^{\circ}$ 1, Vol. 122011.

CARLEY, K. M., LEE, J. S., and KRACKHARDT, D. “Destabilizing Networks”, Connections. Vol. 24, N. ${ }^{\circ}$ 3, 2002. 
COLEMAN, J. "Systems of Social Exchange", Journal of Mathematic Sociology. Vol. 2, 1972.

Cordón Aguilar, J. C. Prueba indiciaria y presunción de inocencia en el proceso penal. San Sebastián, Instituto Vasco de Derecho Procesal, 2012.

DARWIN, Ch. El origen de las especies. Madrid, Alianza, 2003.

DEL ROSAL BLASCO, B. “¿Hacia el Derecho Penal de la postmodernidad?", Revista Electrónica de Ciencia Penal y Criminología. N. ${ }^{\circ}$ 8, 2009.

DUNNE, C., and SHNEIDERMAN, B. "Motif Simplification: Improving Network Visualization Readability with Fan, Connector, and Clique Glyphs", VV. AA. Proceedings of the SIGCHI Conference on Human Factors in Computing Systems. Paris, ACM, 2013.

FREEMAN, L. "A Set of Measures of Centrality Based on Betweenness", Sociometry. Vol. 40, 1977.

GAFISUD. Reunión conjunta de tipologías Gafisud. Quito, GAFISUD, 2012.

GALTUnG, J. Teoría y métodos de investigación social. Buenos Aires, Eudeba, 1966.

GARAY SALAMANCA, L., y SALCEDO-ALBARÁN, E. Redes ilícitas y reconfiguración de Estados. El caso Colombia. Bogotá, Vortex-ICTJ, 2012. 
GRAFE ARIAS, F. Reformas institucionales en materia de seguridad y sistemas judiciales en la lucha contra la impunidad en Iberoamérica. Valencia, Tirant lo Blanch, 2009.

Granovetter, M. "The Strength of Weak Ties". American Journal of Sociology. Vol. 78, N. ${ }^{\circ}$ 6, 1973.

GUZMÁN, N. La verdad en el proceso penal (Una contribución a la epistemología jurídica). Buenos Aires, Editores del Puerto, 2011.

HACKING, I. Representar e intervenir. México, Paidós, 1996.

HANSON, N. Patrones de descubrimiento. Observación y explicación. Madrid, Alianza Editorial, 1985.

JAMES, W. Pragmatismo, un nuevo nombre para algunos antiguos modos de pensar. Barcelona, Orbis, 1984.

JUANES PECES, A. "El principio de presunción de inocencia en la doctrina del Tribunal Constitucional, con especial referencia a si los indicios pueden destruir tal presunción”. Poder Judicial. N. ${ }^{\circ} 6$ esp., 1989.

KLEINROCK, L. "An Early History of Internet", SEEE Communications Magazine. August, 2010.

KORZYBSKI, A. "The Role of Language in the Perceptual Processes", BLAKE, R. and GLENN, V. (eds.). Perception: an Approach to Personality. New York, The Ronald Press Company, 1951. 
KREBS, V. "Mapping Networks of Terrorist Cells", Connections, Vol. 24, N. ${ }^{\circ}$ 3, 2001.

LAUCHS, M., KEAST, R., and CHAMBERLAIN, D. "Resilience of a Corrupt Police Network: the First and Second Jokes in Queensland". Crime, Law and Social Change. Vol. 57, N. ${ }^{\circ} 2,2011$.

LlOBET ANGLí, M. Derecho penal del terrorismo. Límites de su punición en un Estado democrático. Madrid, La Ley, 2010.

LOZARES COLINA, C. "La teoría de redes sociales", Revista de Sociología. v. 48, 1996.

MANDELBROT, B. Los objetos fractales. Madrid, TusQuets, 1987.

MARCET GARCÍA, E., PALACIOS-CALLENDER, M. y MARCET SÁNCHEZ, M. "Diseño y programación de un software de transformación de matrices para el análisis de redes", Redes Revista Hispana para el Análisis de Redes Sociales. v. $27, \mathrm{~N} .^{\circ} 1,2016$.

MARSDEN, P. V. "Restricted Access in Networks and Models of Power", American Journal of Sociology. Vol. 88, N. ${ }^{\circ} 4$, 1984.

MARTÍ, J. DE, and ZENOU, Y. Social Networks. Stockholm, Research Institute of Industrial Economics, 2009.

MICELI, J., ORSI, O. y RODRÍGUEZ GARCÍA, N. ARS y sistema penal. Valencia, Tirant lo Blanch, 2017. 
MonsalVE, S. "John Nash y la teoría de juegos". Lecturas Matemáticas. v. 24, 2003.

MORSELLI, C. Inside Criminal Networks. New York, Springer, 2009.

NeUmann, J., and MORGENSTERN, O. Theory of Games and Economic Behavior. Princeton, Princeton University Press, 1953.

ORSI, O. G. Sistema penal y crimen organizado. Estrategias de aprehensión y criminalización del conflicto. Buenos Aires, Editores del Puerto, 2007a.

. Lavado de dinero de origen delictivo. Buenos Aires, Hammurabi, 2007b.

- Asociaciones ilícitas terroristas y financiación del terrorismo. Buenos Aires, Editores del Puerto, 2008a.

- "Aportes metodológicos a la investigación y prueba de casos complejos", RODRÍGUEZ GARCÍA, N. y RODRÍGUEZ LÓPEZ, F. (coords.). Corrupción y desarrollo. Valencia, Tirant lo Blanch, 2017.

PARRA QUIJANO, J. Tratado de la Prueba Judicial. 6. a edición. Bogotá, Librería Ediciones del Profesional Ltda., 2007.

PÁSTOR AlCOY, F. Prueba de indicios, credibilidad del acusado y presunción de inocencia. Valencia, Tirant lo Blanch, 2003. 
PEIRCE, Ch. Deducción, inducción e hipótesis. Buenos Aires, Aguilar, 1979.

PIAGET, J. Biología y conocimiento. Madrid, Siglo XXI, 1969.

POE, E. Los crímenes de la calle Morgue. Madrid, Valdemar, 1996.

Poveda CRIAdo, M. A. Delitos en la red. Madrid, Fragua, 2015.

REYNoso, C. Redes sociales y complejidad: Modelos interdisciplinarios en la gestión sostenible de la sociedad y la cultura. Buenos Aires, Editorial Sb, 2011.

ROGGERO, P. et SIBERTIN-BLANC, Ch. "Quand des sociologues rencontrent des informaticiens: essai de formalisation des systèmes d'action concrets", Nouvelles Perspectives en Sciences Sociales. N. ${ }^{\circ}$ 3, 2008.

RUGGA RIVA, C. Il premio per la collaborazione processuale. Milán, Giuffrè, 2002.

RUgGiero, V. Delitos de los débiles y de los poderosos. Buenos Aires, Editorial Ad-Hoc. 2005.

RUSSO, V. e ABET, A. La prova indiciaria e il giusto proceso. Napoli, Jovene, 2001.

SABATÉ, J. "Desviación y control en las sociedades avanzadas". Revista de Sociología. v. 28, 1987. 
Sageman, M. Understanding Terror Networks. Philadelphia, University of Pennsylvania Press, 2004.

SAMAJA, J. Epistemología y metodología. Elementos para una teoría de la investigación científica. Buenos Aires, Eudeba, 1993.

SANTA Agreste, S. C., DE MEOC, P., FERRARA, E., and FIUMARA, G. "Network Structure and Resilience of Mafia Syndicates", Information Sciences. Vol. 351, 2015.

SChÜRMAnN, J. Pattern Classification: a Unified View of Statistical and Neural Approaches. Nueva York, Wiley, 1996.

SILVA MELERO, V. Presunciones e indicios en el proceso penal. Madrid, Instituto Editorial Reus, 1944.

SOTOMAYOR ACOSTA, J. "Criminalidad organizada y criminalidad económica: los riesgos de un modelo diferenciado de Derecho Penal", Revista de Estudios de la Justicia. N. ${ }^{\circ} 12,2010$.

StrogatZ, S. The Emerging Science of Spontaneous Order. New York, Hyperion, 2003.

SUÁREZ, J. "Inferencia razonable, probabilidad de verdad y conocimiento más allá de toda duda razonable", Revista de Derecho Principia Iuris. v. 16, 2011.

Sutherland, E. White Collar Crime. New York, Dryden Press, 1949. 
VON BERTALANFFY, L. Teoría general de sistemas. Madrid, Fondo de Cultura Económica, 1976.

WAINWRIGHT, T. Narconomics: How to Run a Drug Cartel. New York, Public Affairs, 2016.

Watanabe, S. Pattern Recognition: Human and Mechanical. Nueva York, Wiley, 1985.

WIENER, N. Cibernética y sociedad. Buenos Aires, Editorial Sudamericana, 1958.

WU, E., CARleton, R., and DAVIES, G. "Discovering BinLaden's Replacement in al-Qaeda, Using Social Network Analysis: A Methodological Investigation", Perspectives on Terrorism. Vol. 8, N. ${ }^{\circ} 1,2014$.

ZAFFARONI, E. "En torno al concepto de crimen organizado", VIRGOLINI, J. E. S., SLOKAR, A. W., y ZAFFARONI, E. (coords.). Nada personal. Ensayos sobre crimen organizado y sistema de justicia. Buenos Aires, Editorial Depalma, 2001. 